\title{
Amorphous metal-hydrides for optical hydrogen sensing: the effect of adding glassy $\mathrm{Ni}-\mathrm{Zr}$ to $\mathrm{Mg}-\mathrm{Ni}-\mathrm{H}$
}

Mercedes Victoria ${ }^{1}$, Ruud J. Westerwaal ${ }^{1}$, Bernard Dam ${ }^{1}$, and Jacobus L.M. van Mechelen ${ }^{2}$ *

${ }^{1}$ Department of Chemical Engineering, Materials for Energy Conversion and Storage, Delft University of Technology, Julianaweg 136, 2628 BL Delft, The Netherlands.

${ }^{2}$ ABB Corporate Research, Segelhofstrasse 1K, 5405 Baden-Dättwil, Switzerland.

dook.vanmechelen@ch.abb.com

\section{Table of Contents}

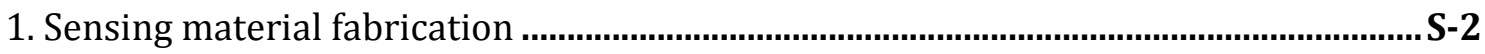

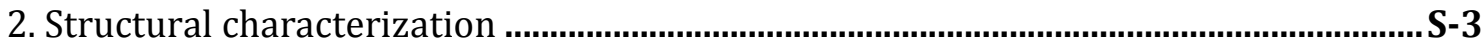

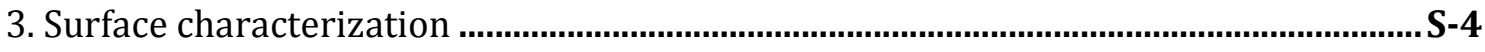

4. Sensor characterization in gas environments ...............................................................

5. Sensor characterization in liquid environments ................................................................. 


\section{Sensing material fabrication}

Mg-Ni-Zr compositional gradient alloy thin films are prepared by co-sputtering at room temperature on quartz substrates of $5 \mathrm{~mm}$ by $70 \mathrm{~mm}$. The deposition is performed using an ultrahigh-vacuum DC/RF magnetron sputtering system (base pressure 10-8 mbar, sputter pressure $3 \times 10^{-3} \mathrm{mbar}$ ). To ensure good adhesion to the substrate, a $3 \mathrm{~nm}$ Ti layer was deposited prior to the sensing layer (see Figure S1). The films are covered in-situ with a 10-30 nm Pd layer to promote $\mathrm{H}_{2}$ dissociation and to prevent oxidation of the underlying sensing film. For measurements in liquid, a $40 \mathrm{~nm}$ PTFE coating was grown on top of the Pd layer by magnetron sputtering in a separate chamber.

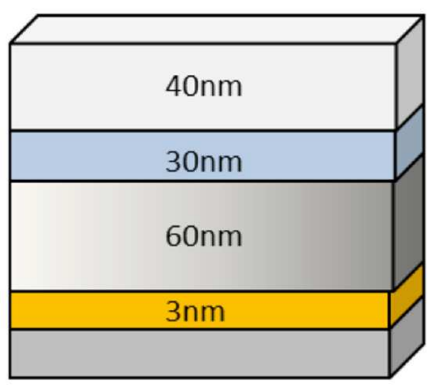

PTFE

$\mathrm{Pd}$

$\mathrm{Mg}-\mathrm{Ni}-\mathrm{Zr}$

$\mathrm{Ti}$

substrate

Figure S1. Schematic representation of the hydrogen sensing material layout. Depending on the purpose of the sample, the substrate material is either a quartz plate or an optical fiber tip.

Following this procedure, $\mathrm{Mg}_{x} \mathrm{Ni}_{100-x-y} \mathrm{Zr}_{y}$ thin films with a compositional gradient $(52<x<64$ and $7<y<29$ ) are deposited. In order to study the effect of Ni-Zr, for each gradient sample the $\mathrm{Mg}$ content is kept almost constant. As a side effect of the deposition technique, the thickness of the film slightly varies from $\sim 60 \mathrm{~nm}$ on the $\mathrm{Zr}$-poor side to $\sim 70 \mathrm{~nm}$ on the $\mathrm{Zr}$-rich side. In previous experiments it was observed that the influence of such a small change in thickness has almost no influence on the hydrogenation behavior. ${ }^{[1]}$

For experiments in oil, Mg-Ni-Zr thin films ( $\sim 60 \mathrm{~nm}$ in thickness) are deposited on the cleaved and coating-free end of glass fibers. The fibers are polyimide coated multimode fibers with 15 $\mu \mathrm{m}$ coating on a $200 \mu \mathrm{m}$ diameter core. Prior to cleavage, the coating has been removed in a reactive way over 1-2 $\mathrm{mm}$ distance from the tip. 


\section{Structural characterization}

Structural characterization has been performed by x-ray diffraction (XRD) using a Bruker D8 Advance equipped with Co source $(\alpha=0.178897 \mathrm{~nm})$. A polymer loading cell enabled in-situ examination at $\sim 0.5$ bar partial hydrogen pressure.

Figure S2.a shows the x-ray diffraction spectra for selected Mg-Ni-Zr thin film compositions in the as-deposited state. Only the Pd (111) and (220) reflections are observed. Figure S2.b shows the diffraction spectra for $\mathrm{Mg}_{52} \mathrm{Ni}_{20} \mathrm{Zr}_{28}$ in the as-deposited state without Pd layer (b.1), in the asdeposited state with Pd layer (b.2) and in the unhydrogenated state after hydrogen cycling (b.3). In the latter two cases the Pd (111) and (220) reflections are also here the only ones that are observed. The increase of the Pd (111) reflection after hydrogen cycling can be explained by a reorientation of the Pd film in [111], resulting in an energetically more favorable packing of Pd. Figure S2.b.4 shows $\mathrm{Mg}_{52} \mathrm{Ni}_{20} \mathrm{Zr}_{28}$ after hydrogen cycling in the hydrogenated state. Here one can observe the $\mathrm{PdH}_{0.7}$ (111) and $\mathrm{PdH}_{0.7}$ (220) reflections. Absence of any Mg-Ni-Zr reflection implies that there is no crystal growth due to the hydrogenation process.

These results indicate amorphousness of the Mg-Ni-Zr thin film structure, in agreement with the glass state of $\mathrm{Ni}-\mathrm{Zr}$ that forms for the $\mathrm{Ni} / \mathrm{Zr}$ ratios considered here.[2]
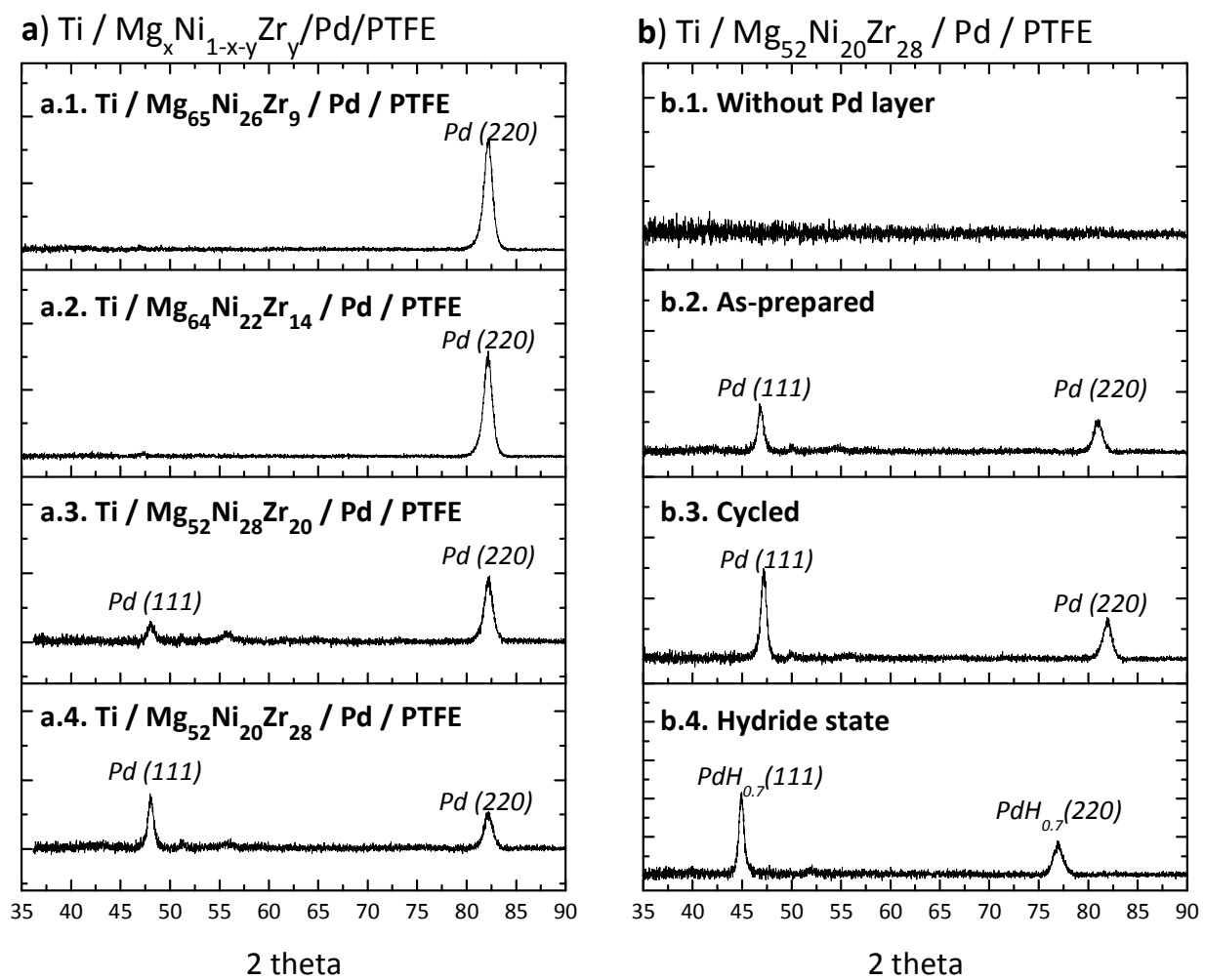

Figure S2. X-ray diffraction spectra for (a) selected Mg-Ni-Zr compositions in the as-deposited state, and (b) $\mathrm{Mg}_{52} \mathrm{Ni}_{20} \mathrm{Zr}_{28}$ without Pd (b.1), with Pd (b.2), after hydrogenation in the unhydrogenated state (b.3), and in the hydrogenated state (b.4). 


\section{Surface characterization}

The surface morphology of Mg-Ni-Zr samples has been studied using Atomic Force Microscopy (AFM). Thin films with different compositions were investigated and gave comparable results. Figure S3 shows the structure on a surface area of $5 \times 5 \mu \mathrm{m}^{2}$ of $\mathrm{Mg}_{60} \mathrm{Ni}_{33} \mathrm{Zr}_{7}$ in the as-deposited state and after 50 hydrogenation cycles. The images evince a very smooth surface with a grain structure of about $10-20 \mathrm{~nm}$. The diagonal pattern is probably due to the polishing quality of the quartz substrate. There is no sign of big clusters of particle formation so it is likely that the film is composed of very small particles. Furthermore, no pin holes, phase segregation, or preferred growth effect, are observed. The right panel in Figure S3 shows that after 50 hydrogenation cycles, the high quality is almost unaltered. Note that on this sample $40 \mathrm{~nm}$ PTFE has been added to the surface, which is probably causing the image to be slightly blurred. However, there is no sign of buckling or segregation, demonstrating the elevated mechanical stability of the sensor material.
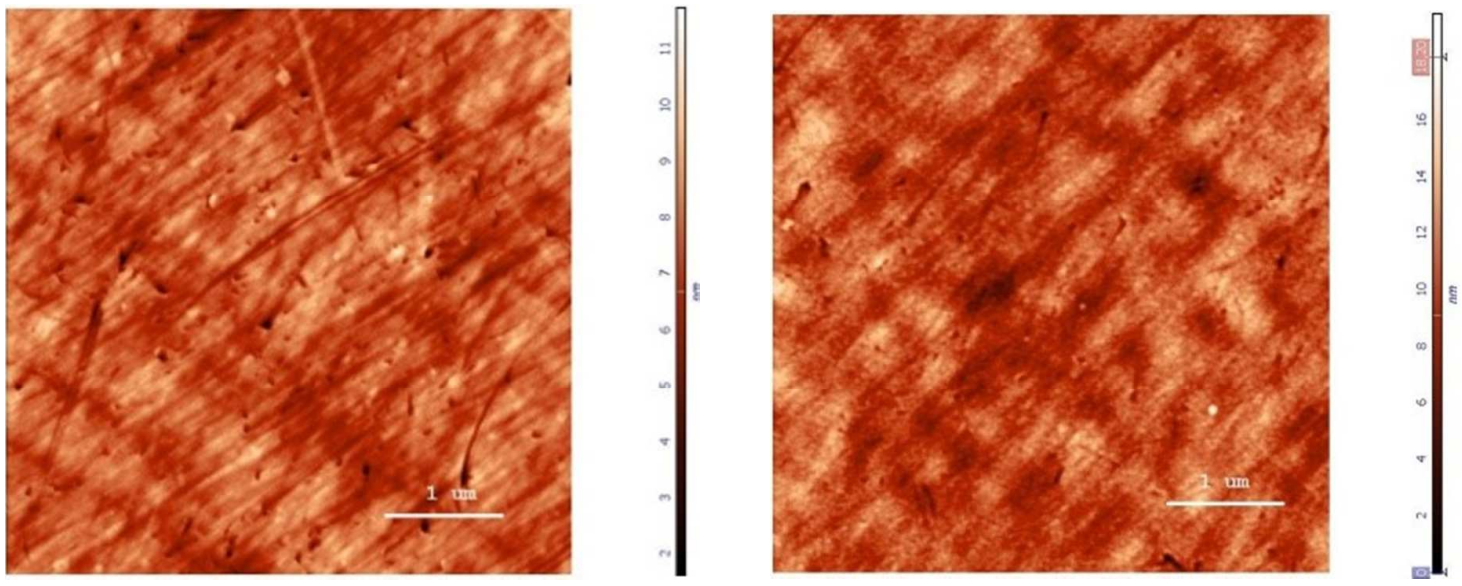

Figure S3. AFM images of $\mathrm{Mg}_{60} \mathrm{Ni}_{33} \mathrm{Zr}_{7}$ covered with $\mathrm{Pd}$ in the as-deposited state (left panel) and after 50 hydrogenation cycles (right panel). The in-plane dimensions are indicated in each image by the white horizontal bar (corresponding to $1 \mu \mathrm{m}$ ). The vertical colour map scale gives the surface height in $\mathrm{nm}$.

\section{Sensor characterization in gas environments}

Optical characterization in gas environments has been performed in a combinatorial way, using so-called hydrogenography. ${ }^{[3]}$ The sensing material stack (see Figure S1) was inserted in a hydrogen loading cell that was illuminated with a $150 \mathrm{~W}$ white light source. The hydrogen pressure inside the cell has been ramped up from $10^{-4}$ mbar to 5 mbar in 19 hours to guarantee equilibrium conditions. Below the cell, a three-channel RGB Sony XC-003 CCD camera continuously monitored the transmitted light intensity in the photon energy range 1.1-3.3 eV. Pressure transmission isotherms (as shown in Figure 1 and Figure S4) have been determined from averaging the three CCD channels. Figure S4 shows the relative transmissivity $\mathcal{T}$ of $\mathrm{Mg}_{52} \mathrm{Ni}_{24} \mathrm{Zr}_{24}$ during absorption and desorption for the $1^{\text {st }}$ and $3^{\text {rd }}$ hydrogenation cycle. After 50 hydrogenation cycles, the absorption curves almost perfectly match (see Figure 1c) and the sensor material manifests a highly reproducible response. 


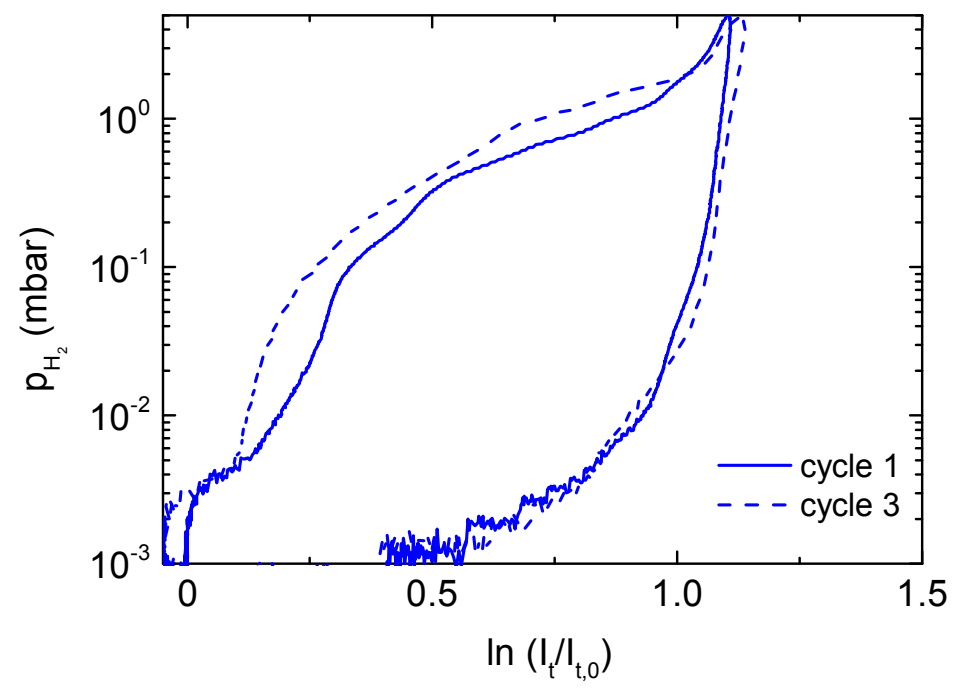

Figure S4. Pressure-transmission isotherms of $\mathrm{Mg}_{52} \mathrm{Ni}_{24} \mathrm{Zr}_{24}$ shown during absorption (upper curves) and desorption (lower curves) for the $1^{\text {st }}$ and $3^{\text {rd }}$ hydrogenation cycle at $21^{\circ} \mathrm{C}$ in a gaseous environment.

In order to map out the temperature dependence of the sensing material, the hydrogen loading cell was put in an oven. Measurements to test repeatability have been performed by repeatedly exposing the sensing material to a mixture of $\mathrm{H}_{2}$ in $\mathrm{Ar}$ and $\mathrm{O}_{2}$ in $\mathrm{Ar}$.

\section{Sensor characterization in liquid environments}

The performance of the sensing material has been tested in liquid environments corresponding to power transformer applications. For this purpose, a fiber optic sensor (using a micro mirror design) has been fabricated as described earlier. A $250 \mathrm{ml}$ oil bath, in direct contact with the ambient, is continuously hydrogenated using a gas disperser with a flow of $250 \mathrm{ml} / \mathrm{min}$. After 15 hydrogenation cycles in gas, the micro mirror sensor has been immersed into the liquid at a distance from the gas insertion to avoid gas bubbles reaching the sensor. The fiber is connected both to a tungsten halogen light source and an Ocean Optics USB2000 CCD-based spectrometer using an optical circulator. The presented data correspond to a wavelength of $635 \mathrm{~nm}$, for which also inexpensive LEDs can be used. Before introducing hydrogen in the bath, the system was pre-flushed with $20 \% \mathrm{O}_{2}$ in Ar to unload the sensor and to assure identical starting conditions. The temperature of the bath was regulated by using water tubes in direct and indirect contact with the bath.

1. Gremaud, R. Hydrogenography: a thin film optical combinatorial study of hydrogen storage materials. Vrije Universiteit Amsterdam, Amsterdam, 2008.

2. Spit, F. H. M.; Drijver, J. W.; Radelaar, S., Hydrogen sorption by the metallic glass Ni64Zr36 and by related crystalline compounds. Scripta Metallurgica 1980, 14 (10), 1071-1076.

3. Gremaud, R.; Broedersz, C. P.; Borsa, D. M.; Borgschulte, A.; Mauron, P.; Schreuders, H.; Rector, J. H.; Dam, B.;

Griessen, R., Hydrogenography: An Optical Combinatorial Method To Find New Light-Weight Hydrogen-Storage

Materials. Advanced Materials 2007, 19, 2813-2817. 